\title{
Evaluation of left ventricular functions with two-dimensional speckle-tracking echocardiography (2D-STE) and N-terminal ProBNP in diabetic children
}

\author{
Ayşe Şimşek ${ }^{1}$, Özlem Turan ${ }^{1}$, Murat Çiftel ${ }^{1}$, Firat Kardelen ${ }^{1}$, Erdem Durmaz ${ }^{2}$, Sebahat \\ Özdem ${ }^{3}$, Gayaz Akçurin ${ }^{1}$, Halil Ertuğ ${ }^{1}$ \\ Departments of ${ }^{1}$ Pediatric Cardiology, ${ }^{2}$ Endocrinology and ${ }^{3}$ Biochemistry, Akdeniz University Faculty of Medicine, Antalya, \\ Turkey.E-mail: draysesimsek@hotmail.com
}

Received: 12th September 2017, Accepted: 6th March 2018

SUMMARY: Şimşek A, Turan Ö, Çiftel M, Kardelen F, Durmaz E, Özdem $S$, Akçurin G, Ertuğ H. Evaluation of left ventricular functions with twodimensional speckle-tracking echocardiography (2D-STE) and $\mathrm{N}$-terminal ProBNP in diabetic children. Turk J Pediatr 2018; 60: 633-641.

The purpose of this study was to examine the existence of subclinical left ventricular dysfunction by using 2D-STE and NT-ProBNP levels in children and adolescent patients with type 1 diabetes mellitus. Furthermore, it was also aimed to investigate the effects of the diabetes duration and the metabolic control of the disease on cardiac functions.

The patient group was composed of 63 children who were being followed up for the type 1 diabetes mellitus. The control group was composed of 36 healthy children who were of the similar age. Patients with type 1 diabetes mellitus were divided into groups; according to the duration of the disease; group 1: 3-5 years, group 2: 6-10 years of follow-up. The conventional echocardiography and 2D-STE were applied to all of the patients and control individuals. NT-Pro BNP level was measured in the diabetes group.

In the conventional echocardiographic examination; there was no difference between the patient and control groups in terms of left ventricular systolic functions, left ventricular diastolic functions; late-diastolic flow velocity in mitral valve (A) values increased and E-wave/A-wave ratio (E/A ) values decreased in diabetes mellitus patients. According to the 2D-STE results; global longitudinal strain, $(-17.28 \pm 2.24 v s .-19.49 \pm 2.22 ; \mathrm{p}<0.05)$ and circumferential strain $(-12.86 \pm 3.19$ vs. $-17.71 \pm 4.62$; $\mathrm{p}<0.05)$ were lower in diabetic patients compared to the parameters of control group individuals. There was no difference between levels of NT-ProBNP of the group 1 and group 2 diabetes mellitus patients.

Our study showed that there was a dysfunction on the left ventricular systolic functions of the patients with type 1 diabetes mellitus. NT-Pro BNP levels were not considered as a distinguishing factor for the early stages of diabetes mellitus.

Key words: diabetes mellitus type 1, strain, NT- brain natriuretic peptide, left ventricular dysfunction.

One of the most important causes of morbidity and mortality are cardiovascular complications in patients with type 1 diabetes mellitus. ${ }^{1-2}$ Diabetes mellitus causes changes in the cardiac structure and its functions which lead to diabetic cardiomyopathy. ${ }^{3-4}$ The pathogenesis of the diabetic cardiomyopathy is believed to be multifactorial and even though the exact reason has not yet been known, hyperglycemia is thought to play a key role. Pathogenic changes 
may affect the structure of the heart and they can lead to myocardial fibrosis. ${ }^{5}$ Myocardial fibrosis in diabetes mellitus eventually causes the diastolic and systolic dysfunction of the left ventricle. ${ }^{6}$

Echocardiography is the most fundamental technique that is used in the diagnosis of the diabetic cardiomyopathy. In the early stages of diabetic cardiomyopathy, left ventricular ejection fraction (EF) is preserved and diastolic function becomes abnormal. ${ }^{7}$ Furthermore, left ventricular -EF measurement is not an objective tool for the evaluation of left ventricular systolic functions. Conventional echocardiography has some limitations in revealing subclinical left ventricular systolic dysfunction.

In recent years, left ventricular systolic functions have been analyzed by using 2D-STE even during the subclinical period. ${ }^{8}$ Although there are many studies suggesting how structural and functional changes in the myocardium can lead to the development of heart failure in adult diabetes mellitus patients ${ }^{9-11}$, only a limited number of them have examined the status of pediatric diabetes mellitus patients. ${ }^{12-13}$

B-type natriuretic peptide (BNP) is a polypeptide neuro-hormone and it is secreted from the atrial and particularly from ventricular myocytes ${ }^{14}$. $\mathrm{BNP}$ is produced as a prohormone; pro BNP (108 amino acids), which is cleaved by furin (27) to form the active BNP (77-108) and the inactive NT-BNP (1-76 molecules). It has been reported that BNP and NT-BNP are significantly correlated with each other and also with left ventricular -EF. ${ }^{15}$ However, the increase in NTBNP level is much greater (4-fold) than BNP levels in subjects with moderate left ventricular dysfunction. NT-ProBNP neutral endopeptidase level is not affected. The half-life of NT-ProBNP is longer and its measurement is easier. Because of its uncomplicated measurement method, NTProBNP levels are more frequently tested for the diagnosis of heart failure and its follow-up. ${ }^{16}$

The aim of this study was to investigate the presence of subclinical left ventricular systolic dysfunction by using the 2D-STE method and NT-Pro BNP levels, as an alternative to conventional echocardiographic examination in pediatric type 1 diabetes mellitus patients who were followed up for not less than three years. Besides, it was also aimed to investigate the effects of the diabetes duration and the metabolic control of the disease on cardiac functions.

\section{Material and Methods}

\section{Study population}

The study was conducted with 63 patients (7-18 years) who did not have micro- and macrovascular complications and who were followed up after a diagnosis of type 1 diabetes mellitus at the Pediatric Endocrinology Department. All members of the patient group were given an insulin regimen. There were no clinical symptoms indicating the cardiac dysfunction. Patients with diabetes mellitus were divided into two groups according to the duration of their follow-ups. Group 1: followed-up for three to five years and Group 2; followed-up for six to ten years with type1 diabetes mellitus.

The control group was composed of 36 healthy individuals (6-17 years) who were similar to the patient group in terms of their age. The control group showed the normal physical examination, normal echocardiographic results, and they did not have any other systemic disease. They were admitted to the Pediatric Cardiology Clinic with complaints of chest pain or murmur.

Medical histories of all individuals were obtained and physical examinations were performed for all subjects. The age, weight, height, and systolic blood pressure (SBP), diastolic blood pressure (DBP) measurements of all individuals were recorded. BMI was calculated as body weight $(\mathrm{kg})$ divided by square of height $\left(\mathrm{m}^{2}\right)$. We used population-specific data for calculation of the anthropometric values to define as standard deviation score (SDS). ${ }^{17}$

The mean glycated hemoglobin (HbAlc) level of typel diabetes mellitus patients was determined by averaging the lowest $4 \mathrm{HbAlc}$ levels that had been measured over the previous two years.

Patients who had developed microvascular complications; including nephropathy, retinopathy or neuropathy and concurrent systemic diseases were excluded from the study.

The Local Ethical Committee of Akdeniz University Faculty of Medicine approved the study (Project number: 2012.04.0103.009). Informed consent were obtained from all patients and their families. 


\section{Conventional echocardiography}

All of the patients and control subjects underwent a detailed echocardiographic examination. Echocardiographic assessments were performed by using simultaneous electrocardiographic recordings with the help of the Vivid 7 Pro ultrasound system (GE Medical Systems, Horten, Norway) by using appropriate transducers. Left ventricular systolic functions were assessed by using the M-mode echocardiography in parasternal long axis view. The left ventricular internal diameters at the end-systole (LVIDs) and end-diastole (LVIDd), fractional shortening (FS), ejection fraction (EF) values were measured.

To determine left ventricular diastolic functions; the mitral inflow signal was acquired in an apical four-chamber view. Doppler echocardiographic examinations were used to obtain images of the early diastolic flow (E) and late diastolic flow (A) wave velocities of the mitral valve. These were then employed to calculate the $\mathrm{E} / \mathrm{A}$ ratio.

\section{NT-ProBNP (N-terminal pro brain natriuretic peptide)}

Blood samples which were obtained from type1 diabetes mellitus patients were collected in the gel-filled biochemistry tubes. Samples were centrifuged at 4,000 rpm for five minutes. Upon centrifugation, the sera were separated and samples were preserved at $-80^{\circ} \mathrm{C}$ until analysis. The serum samples were studied at the Central Laboratory of the Department of Medical Biochemistry in order to identify the NT-pro BNP concentration $(\mathrm{pg} / \mathrm{ml})$. The concentrations of NT-proBNP in the serum were assessed by using the Cobas 8000 automated analyzer.

\section{Two-dimensional speckle-tracking echocardiography (2D-STE)}

2D-STE examinations were performed with all of the subjects in both patient and control groups. Standard grey scale $2 \mathrm{D}$ images were obtained at a rate of $60-80$ frames/s from the apical -four chamber, -three chamber, -two chamber and the parasternal short-axis views at the papillary muscle level of the left ventricle. The images were loaded into a CD in the cine loop format and then analyzed by using an
Echo Pac (software version 8, GE, Healthcare) program. The analysis of the image recordings and the endocardial borders on the basis of successive points on a single frame were all conducted by the same practitioner.

Longitudinal strain peak $S$ values were measured by using the apical 4, 3, 2 chamber images and followed by averaging the three chambers and the determination of the global longitudinal strain value. The average values of circumferential peak $G$ and radial peak $G$ measurements were obtained, and the global circumferential strain value and global radial strain values identified, by employing the measurements obtained from the parasternal short-axis views at the level of the papillary muscle.

\section{Statistical analysis}

The statistical analysis was performed by using the Statistical Package for Social Sciences (SPSS; SPSS, Inc., Chicago, IL, USA) version 18.0 for Windows. Variables were expressed as mean \pm standard deviation (SD). Student- $t$ test was used in order to determine the statistical significance between parametric variables whereas Mann-Whitney $U$ test was used to determine the statistical significance between non-parametric variables. Furthermore, Kruskal Wallis test was used to compare three groups. In the evaluation of relationships between parameters, Pearson correlation analysis was used for normally distributed parameters and Spearman's rho correlation analysis was used in case parameters were not normally distributed. Statistical significance was accepted when $\mathrm{p}$ value was lower than $0.05(\mathrm{p}<0.05)$.

\section{Results}

Totally, 63 patients and 36 healthy controls were included in the study. The type1 diabetes mellitus patients were divided into two groups on the basis of their follow-up durations. Accordingly, Group 1 was composed of 32 type 1 diabetes mellitus patients who were followed up for a period of three to 5 years and Group 2 was composed of 31 type 1 diabetes mellitus patients who were followed up for a period of six to 10 years.

The patient group had a mean age of $13.8 \pm 2.9$ years. There were 30 male $(48 \%)$ and 33 
Table I. Demographic and Laboratory Variables of the Patients and Controls.

\begin{tabular}{|c|c|c|c|c|}
\hline \multirow[b]{2}{*}{ Variables } & \multicolumn{2}{|c|}{ Patient Groups } & \multirow{2}{*}{$\begin{array}{l}\text { Controls } \\
(\mathrm{N}=36)\end{array}$} & \multirow[b]{2}{*}{$\mathrm{P}$} \\
\hline & $\begin{array}{c}\text { Group } 1 \quad(\mathrm{~N}=32) \\
\text { Duration: } 3-5 \text { years }\end{array}$ & $\begin{array}{c}\text { Group } 1 \quad(\mathrm{~N}=31) \\
\text { Duration: } 6-10 \text { years }\end{array}$ & & \\
\hline Follow-up duration, years & $4.07 \pm 0.72$ & $7.50 \pm 1.41$ & $0.0 \pm 0.0$ & $<0.05$ \\
\hline Age, years & $13.53 \pm 2.95$ & $14.10 \pm 2.89$ & $12.42 \pm 3.45$ & NS \\
\hline Female / Male & $17 / 15$ & $16 / 15$ & $17 / 19$ & NS \\
\hline BMI- SDS & $-0.5 \pm 1.13$ & $0.06 \pm 1.23$ & $-0.2 \pm 1.18$ & NS \\
\hline SBP, mm Hg & $105 \pm 12$ & $108 \pm 15$ & $100 \pm 10$ & NS \\
\hline DBP, mm Hg & $68 \pm 9$ & $70 \pm 10$ & $65 \pm 8$ & NS \\
\hline HbAlc, \% & $9.80 \pm 2.04$ & $9.38 \pm 1.9$ & - & NS \\
\hline
\end{tabular}

BMI-SDS: body mass index-standard deviation score, DBP: diastolic blood pressure, HbA1c: glycated hemoglobin NS: non-significant, SBP: systolic blood pressure,

female $(52 \%)$ patients. On the other hand, the mean age of the control group individuals was $12.4 \pm 3.4$ years and there were 17 male $(47 \%)$ and 19 female (53\%) subjects. Mean HbAlc of the patient groups was $9.5 \pm 1.9$.

There was no statistically significant difference between the patient and control groups in terms of age, sex, SBP, DBP and BMI-SDS. There was no statistically significant difference between group 1 and group 2 in terms of HbAlc levels $(\mathrm{p}>0.05)$. The demographic and laboratory variables of the groups are shown in Table I.

\section{Results of conventional echocardiography}

Left ventricular systolic functions did not show any statistically significant difference between the patient and control groups in terms of EF, LVIDs, LVIDd, FS values.

Patient and control groups were compared to each other in terms of parameters indicating LV diastolic functions: mitral valve E-wave velocity,
A-wave velocity, and E/A ratios. Mitral A wave velocity value $(0.58 \pm 0.14$ vs. $0.50 \pm 0.11$; $\mathrm{p}<0.05)]$ was higher, and $\mathrm{E} / \mathrm{A}$ ratio $(1.76 \pm 0.37$ vs. $2.11 \pm 0.34 ; p<0.05)]$ was lower in type 1 diabetes mellitus patients compared to the control group. Diastolic function parameters such as mitral E-wave, A-wave and $\mathrm{E} / \mathrm{A}$ values were within the normal reference range in all groups according to the age groups. ${ }^{18}$ The results of the conventional echocardiography are shown in Table II.

Systolic and diastolic functions were similar between Group 1 and Group 2 (Table III).

\section{Results of 2D-STE echocardiography}

Longitudinal left ventricular deformation parameter: Global longitudinal strain $(-17.28 \pm 2.2 .4$ vs. $-19.49 \pm 2.22 ; \mathrm{p}<0.05)$ was significantly lower in diabetes mellitus patients compared the control group and reference range according to the age groups. ${ }^{19}$

Table II. Conventional Echocardiographic Measurements of the Patients and Controls.

\begin{tabular}{cccc}
\hline Measurements & Patients $(\mathrm{N}=63)$ & Controls $(\mathrm{N}=36)$ & $\mathrm{P}$ \\
\hline EF, \% & $73.84 \pm 4.93$ & $75.05 \pm 5.09$ & NS \\
FS, \% & $42.68 \pm 4.43$ & $43.66 \pm 4.8$ & NS \\
LVIDd, cm & $4.21 \pm 0.44$ & $4.08 \pm 0.47$ & NS \\
LVIDs, cm & $2.41 \pm 0.28$ & $2.18 \pm 0.26$ & NS \\
E, m/sec & $0.99 \pm 0.14$ & $1.04 \pm 0.17$ & NS \\
A, m/sec & $0.58 \pm 0.14$ & $0.50 \pm 0.11$ & $<0.05$ \\
E/A ratio & $1.76 \pm 0.37$ & $2.11 \pm 0.34$ & $<0.05$
\end{tabular}

A: late-diastolic flow velocity in mitral valve, E: early-diastolic flow velocity in mitral valve, E/A: E-wave/A-wave ratio, EF: Ejection fraction, FS: Fractional shortening, LVIDd: left ventricular end-diastolic diameter, LVIDs: left ventricular end-systolic diameter, NS: non-significant. 
Table III. Conventional Echocardiographic Measurements in Patients with Type-1 Diabetes Mellitus According to Disease Duration.

\begin{tabular}{|c|c|c|c|}
\hline \multirow[b]{2}{*}{ Measurements } & \multicolumn{2}{|c|}{ Disease Duration } & \multirow[b]{2}{*}{$\mathrm{P}$} \\
\hline & $3-5$ years $(\mathrm{N}=32)$ & $6-10$ years $(\mathrm{N}=31)$ & \\
\hline $\mathrm{EF}, \%$ & $75 \pm 4.7$ & $72 \pm 4.9$ & NS \\
\hline FS, \% & $43.7 \pm 4.5$ & $41.6 \pm 4.16$ & NS \\
\hline LVIDd, $\mathrm{cm}$ & $4.16 \pm 0.45$ & $4.26 \pm 0.43$ & NS \\
\hline LVIDs, cm & $2.34 \pm 0.28$ & $2.48 \pm 0.27$ & NS \\
\hline $\mathrm{E}, \mathrm{m} / \mathrm{sec}$ & $0.99 \pm 0.13$ & $0.98 \pm 0.15$ & NS \\
\hline $\mathrm{A}, \mathrm{m} / \mathrm{sec}$ & $0.59 \pm 0.16$ & $0.57 \pm 0.12$ & NS \\
\hline $\mathrm{E} / \mathrm{A}$ ratio & $1.77 \pm 0.42$ & $1.76 \pm 0.31$ & NS \\
\hline
\end{tabular}

A: late-diastolic flow velocity in mitral valve, E: early-diastolic flow velocity in mitral valve, E/A: E-wave/A-wave ratio, EF: Ejection fraction, FS: Fractional shortening, LVIDd: left ventricular end-diastolic diameter, LVIDs: left ventricular end-systolic diameter, NS: non-significant.

Circumferential left ventricular deformation parameter: Circumferential strain $(-12.86 \pm 3.19$ vs. $-17.71 \pm 4.62 ; \mathrm{p}<0.05)$ was significantly lower in diabetes mellitus patients compared the control group and reference range. ${ }^{19}$

Radial strain $(36.74 \pm 16.75$ vs. $42.86 \pm 13.31$; $\mathrm{p}>0.05)$ value was not significantly different between diabetes mellitus patients and control group individuals.

Results of the 2D-STE echocardiography measurements are shown in Table IV. Strain values were similar between Group 1 and group $\mathrm{G}$ patients (Table V). Ventricular longitudinal strain measurements showed negative correlation with LVIDd $(\mathrm{r}=-0.28, \mathrm{p}<0.05)$ and LVIDs $(r=-0.25, p<0.05)$ measurements. Ventricular circumferential strain showed negative correlation with LVIDd $(r=-0.26$, $p<0.05)$. Ventricular radial strain showed negative correlation with LVIDs $(r=-0.26$, $\mathrm{p}<0.05)$ and positive correlation with E-wave $(\mathrm{r}=0.26, \mathrm{p}<0.05)$.

\section{Results of NT- proBNP (N-terminal pro brain natriuretic peptide)}

NT-proBNP levels were identified in the group of 63 type $1 \mathrm{DM}$ patients. All patients were within the normal age range ${ }^{15}$. There was no statistically significant difference between two patient groups in terms of NT-ProBNP ( $p>0.05)$ whereas the average value was higher in the group with longer follow-up.

Group 1: $34.43 \pm 27.7 \mathrm{pg} / \mathrm{ml}$ (min: 51; max: $136 \mathrm{pg} / \mathrm{ml}$ )

Group 2: $41.65 \pm 36.42 \mathrm{pg} / \mathrm{ml}$ (min: 7; max: $180 \mathrm{pg} / \mathrm{ml}$ )

There was a negative correlation between NTPro BNP levels and age $(r h o=-0.55, p<0.05)$, ventricular longitudinal strain $(\mathrm{rho}=-0.22 \mathrm{p}<$ 0.05 ), circumferential strain (rho $=-0.25, \mathrm{p}<$ $0.05)$. There was a positive correlation between NT-Pro BNP levels and LVIDd (rho $=0.27$, $\mathrm{p}<0.05)$ and LVIDs $(\mathrm{rho}=0.25, \mathrm{p}<0.05)$ diameters.

\section{Glycemic control}

The mean HbAlc value of diabetic patients who were followed up for three to five years was $9.80 \pm 2.04 \%$; and it was $9.38 \pm 1.9 \%$ in the group of diabetic patients who were followed up for 5 to 10 years $(p>0.05)$.

Left ventricular systolic function: EF, FS, LVIDd, LVIDs, left ventricular diastolic function $\mathrm{E}$ wave, E/A ratio, A wave, longitudinal strain, radial strain, and circumferential strain values were compared between diabetes mellitus patients with HbA1C $<7.5$ and diabetes mellitus patients with $\mathrm{HbA} 1 \mathrm{c}>7.5$. No statistical significance was detected between these patient 
Table IV. Two-Dimensional Speckle Tracking Echocardiography Measurements of the Patients and Controls.

\begin{tabular}{|c|c|c|c|}
\hline Measurements & Patients $(\mathrm{N}=63)$ & Controls $(\mathrm{N}=36)$ & $\mathrm{P}$ \\
\hline Longitudinal strain, \% & $-17.28 \pm 2.24$ & $-19.49 \pm 2.22$ & $<0.05$ \\
\hline Radial strain , \% & $36.74 \pm 16.75$ & $42.86 \pm 13.31$ & NS \\
\hline Circumferential strain, \% & $-12.86 \pm 3.19$ & $-17.71 \pm 4.62$ & $<0.05$ \\
\hline
\end{tabular}

NS: non-significant.

Table V. Two-Dimensional Speckle-Tracking Echocardiography Measurements in Patients with Type-1 Diabetes Mellitus According to Disease Duration.

\begin{tabular}{|c|c|c|c|}
\hline \multirow[b]{2}{*}{ Measurements } & \multicolumn{2}{|c|}{ Disease Duration } & \multirow[b]{2}{*}{$\mathrm{P}$} \\
\hline & $3-5$ years $(N=32)$ & $6-10$ years $(\mathrm{N}=31)$ & \\
\hline Longitudinal strain, \% & $-17.19 \pm 2.26$ & $-17.37 \pm 2.26$ & NS \\
\hline Radial strain , \% & $34.64 \pm 13.89$ & $38.90 \pm 19.26$ & NS \\
\hline Circumferential strain, \% & $-12.63 \pm 2.94$ & $-13.10 \pm 3.46$ & NS \\
\hline
\end{tabular}

groups.

HbAlc level showed a positive correlation with A-wave velocity $(\mathrm{r}=0.25, \mathrm{p}<0.05)$ and negative correlation with the $\mathrm{E} / \mathrm{A}$ ratio $(\mathrm{r}=-$ $0.24, \mathrm{p}<0.05)$.

\section{Duration of DM}

There was no correlation between follow-up duration and the results of the conventional echocardiography, left ventricular strain results, and NT-Pro BNP levels.

\section{Discussion}

Epidemiological studies have revealed that a prevalence of heart failure is even high in diabetes mellitus patients with normal left ventricular systolic functions. ${ }^{20}$ Therefore, detecting diabetic cardiomyopathy in the early stage is important in order to prevent the heart failure.

The spectrum of diabetic heart disease involves a progression of a normal heart towards preclinical left ventricular diastolic and systolic dysfunction which is further followed by the overt echocardiographic evidence of left ventricular dysfunction and finally symptomatic heart failure. ${ }^{5}$ Left ventricular diastolic dysfunction, as described in the early stages of the disease, can be observed in diabetes mellitus patients at the time they have preserved
EF. ${ }^{7,13}$ In our study, according to the results of conventional echocardiographic examinations, mitral valve $\mathrm{A}$-wave increased and $\mathrm{E} / \mathrm{A}$ ratio decreased in the diabetic group, even though the diastolic function was within the normal reference range for all cases. ${ }^{18}$ This finding indicates the initiation of the left ventricular diastolic dysfunction in diabetic patients. Left ventricular systolic functions such as EF, FS, LVIDd, and LVIDs were observed in the normal reference range ${ }^{18}$ in all the patients and there was no difference between groups.

As specified in previous studies, the recently introduced 2D-STE method is more sensitive than the conventional echocardiography in detecting the subclinical ventricular dysfunction in various clinical disorders. ${ }^{21}$ The 2D-STE method provides a simple and angleindependent evaluation of left ventricular deformation in the longitudinal, radial, and circumferential directions. ${ }^{22}$ STE can be used to compare tissue Doppler images because it enables the elimination of angle dependency and the need for high frame rates, and allows echocardiographic measurement of radial and circumferential strains. Moreover, when compared with magnetic resonance imaging as a bedside tool, it is a cheap and readily available procedure. ${ }^{23}$ According to our results, left ventricular strain values in diabetes mellitus patients were lower than the 
reference values according to the age groups. ${ }^{19}$ Furthermore, left ventricular strain values in diabetes mellitus patients were lower compared to control individuals. This shows that systolic dysfunction starts at an early stage in diabetic patients.

Other relevant studies also reported that systolic dysfunction starts in longitudinal functions. ${ }^{12,24}$ Our findings were in line with results of these studies. In our study, we found that LV longitudinal strain values were significantly lower in diabetic patients compared to the control group individuals. This shows us that the cardiac involvement induced by diabetes begins during childhood.

In our study, the NT-proBNP levels in type1 diabetic pediatric patients were within the normal range. ${ }^{15}$ Previous studies have reported that the NT-pro BNP level increased with the state of left ventricular dysfunction ${ }^{25}$ and it was in correlation with the diastolic dysfunction. ${ }^{26}$ In our study, we demonstrated a positive correlation between NT-pro BNP levels and left ventricular diameters. However, most of the studies in the literature included adult subjects and studies in pediatric population are limited.

Currently there are no studies addressing the correlation between NT-proBNP levels and left ventricular strain measurements in children diagnosed with typel diabetes mellitus. In our study, it was shown that there was a negative correlation between NT-proBNP levels and left ventricular global longitudinal strain and circumferential strain values in pediatric DM patients. These findings show that there is a relationship between NT-Pro BNP levels and left ventricular systolic functions. Ersboll $\mathrm{M}$ et al. ${ }^{27}$ examined the relationship between NT- ProBNP levels and left ventricular global longitudinal strain in adult patients with myocardial infarcts. The global longitudinal strain values obtained by speckle-tracking analysis were found to have a significant correlation with NT-proBNP, and had a moderate correlation with ejection fractions.

It has been reported that hyperglycemia and glycosylation products are the factors responsible for the development of left ventricular systolic (specifically long-axis) and diastolic dysfunction in diabetic patients. $9,28-30$ The accumulation of glycosylation products and changes in the myocardial calcium metabolism result in the endothelial dysfunction, microvascular damage, and finally decreased ventricular compliance and relaxation..$^{7-26}$ The extent and frequency of diastolic dysfunction was directly proportional to the HbA1c levels. In our study, we observed a correlation between HbA1c levels and the diastolic dysfunction. Another study conducted with the typel diabetes mellitus pediatric patients also indicated a correlation between HbA1c levels and the diastolic dysfunction. ${ }^{9}$ [9]

We found that follow-up duration did not have any effect on the cardiac functions of type 1 diabetic patients. According to the results of conventional echocardiography and the 2D-STE methods, no difference was identified between the left ventricular systolic and diastolic functions of patients who were followed up for three to five years and six to 10 years. Studies in adults reported that the duration of diabetes was the only independent determining factor of the reduced global longitudinal strain and diastolic function abnormalities. ${ }^{10,31} \mathrm{At}$ the same time, no significant difference was found between patient groups in terms of NT-Pro BNP levels, whereas the NT-Pro BNP levels were higher in the longer followed-up group. One reason for these findings can be that $\mathrm{HbA1c}$ levels were similar between groups and cardiac dysfunction was also associated with the glycemic level in addition to the follow-up duration.

Our study had several limitations. For instance, we could not perform the tissue Doppler echocardiography in order to investigate the diastolic dysfunction. Our study was an observational study and did not include the clinical follow-up and exercise capacities of the patients. Furthermore, no evaluation has been made in terms of the relationship between the results and the cardiovascular prognosis.

In conclusion, conventional echocardiography may be insufficient in showing left ventricular systolic dysfunction in the early stage of type 1 diabetes mellitus. We detected deteriorations in left ventricular systolic functions with 2D-STE methods in type 1 diabetes mellitus patients while conventional echocardiographic examinations were in normal range. We determined that the NT-Pro BNP level was not a useful tool to differentiate cardiac dysfunction during early stages of diabetes. Metabolic 
control was important in the determination of the diastolic function. The early detection of these effects on target organs may prevent the irreversible damages. The early identification of complications and the implementation of protective measures are important for the improvement in the quality of life and for the prevention of morbidity and mortality.

\section{REFERENCES}

1. Kannel WB, McGee DL. Diabetes and cardiovascular disease. The Framingham study. JAMA 1979; 241: 2035-2038.

2. Maisch B, Alter P, Pankuweit S. Diabetic cardiomyopathyfact or fiction? Herz 2011; 36: 102-115.

3. Karagöz A, Bezgin T, Kutlutürk I, et al. Subclinical left ventricular systolic dysfunction in diabetic patients and its association with retinopathy: A 2D speckle tracking echocardiography study. Herz 2015; 40 (Suppl 3): $240-246$

4. Fang ZY, Prins JB, Marwick TH. Diabetic cardiomyopathy: Evidence, mechanisms and therapeutic implications. Endocr Rev 2004; 25: 543-567.

5. Aneja A, Tang WH, Bansilal S, Garcia MJ, Farkouh ME. Diabetic cardiomyopathy: Insights into pathogenesis, diagnostic challenges, and therapeutic options. Am J Med 2008; 121: 748-757.

6. Turkbey EB, Backlund JY, Genuth S, et al. DCCT/ EDIC Research Group. Myocardial structure, function, and scar in patients with type 1 diabetes mellitus. Circulation 2011; 124: 1737-1746.

7. Galderisi M. Diastolic dysfunction and diabetic cardiomyopathy: Evaluation by Doppler echocardiography. J Am Coll Cardiol 2006; 48: 15481551.

8. Biswas M, Sudhakar S, Nanda NC, et al. Two-and threedimensional speckle tracking echocardiography: Clinical applications and future directions. Echocardiography 2013; 30: 88-105.

9. Kim EH, Kim YH. Left ventricular function in children and adolescents with type 1 diabetes mellitus. Korean Circ J 2010; 40: 125-130.

10. Di Cori A, Di Bello V, Miccoli R, et al. Left ventricular function in normotensive young adults with wellcontrolled type 1 diabetes mellitus. Am J Cardiol 2007; 99: 84-90.

11. de Simone G, Mureddu GF, Vaccaro O, et al. Cardiac abnormalities in type 1 diabetes. Ital Heart J 2000; 1: 493-499.

12. Altun G, Babaoğlu K, Binnetoğlu K, Özsu E, Yeşiltepe Mutlu RG, Hatun Ş. Subclinical left ventricular longitudinal and radial systolic dysfunction in children and adolescents with type 1 diabetes mellitus. Echocardiography 2016; 33: 1032-1039.

13. Hensel KO, Grimmer F, Roskopf M, Jenke AC, Wirth S, Heusch A. Subclinical alterations of cardiac mechanics present early in the course of pediatric type 1 diabetes mellitus: A prospective blinded speckle tracking stress echocardiography Study. J Diabetes Res 2016; 2016: 2583747.

14. Baxter GF. The natriuretic peptides. Basic Res Cardiol 2004; 99: 71-75.

15. Nir A, Lindinger A, Rauh M, et al. NT-pro-B-type natriuretic peptide in infants and children: Reference values based on combined data from four studies. Pediatr Cardiol 2009; 30: 3-8.

16. Ruskoaho H. Cardiac hormones as diagnostic tools in heart failure. Endocr Rev 2003; 24: 341-356.

17. Neyzi O, Bundak R, Gökçay G, et al. Reference values for weight, height, head circumference, and body mass index in Turkish children. J Clin Res Pediatr Endocrinol 2015; 7: 280-293.

18. Frommelt PC. Diastolic Ventricular Function Assessment. Lai WW, Mertens LL, Geva T, Cohen MS (eds). Echocardiography in Pediatric and Congenital Heart Disease: From Fetus to Adult. Milwaukee: WileyBlackwell, 2010: 76-116.

19. Levy PT, Machefsky A, Sanchez AA, et al. Reference ranges of left ventricular strain measures by twodimensional speckle-tracking echocardiography in children: A systematic review and meta-analysis. J Am Soc Echocardiogr 2016; 29: 209-225.e6.

20. Redfield MM, Jacobsen SJ, Burnett JC Jr, Mahoney DW, Bailey KR, Rodeheffer RJ. Burden of systolic and diastolic ventricular dysfunction in the community: Appreciating the scope of the heart failure epidemic. JAMA 2003; 289: 194-202.

21. Leung DY, Ng AC. Emerging clinical role of strain imaging in echocardiography. Heart Lung Circ 2010; 19: 161-174.

22. Altekin RE, Caglar B, Karakas MS, Ozel D, Deger N, Demir I. Evaluation of subclinical left ventricular systolic dysfunction using two-dimensional speckletracking echocardiography in patients with nonalcoholic cirrhosis. Hellenic J Cardiol 2014; 55: 402-410.

23. Marwick TH. Measurement of strain and strain rate by echocardiography: Ready for prime time? J Am Coll Cardiol 2006; 47: 1313-1327.

24. Andersen NH, Poulsen SH. Evaluation of the longitudinal contraction of the left ventricle in normal subjects by Doppler tissue tracking and strain rate. J Am Soc Echocardiogr 2003; 16: 716-723.

25. Yazıcı D, Yavuz DG, Toprak A, Deyneli O, Akalin S. Impaired diastolic function and elevated NT-proBNP levels in type 1 diabetic patients without overt cardiovascular disease. Acta Diabetol 2013; 50: 155161.

26. Salem M, El Behery S, Adly A, Khalil D, El Hadidi E. Early predictors of myocardial disease in children and adolescents with type 1 diabetes mellitus. Pediatr Diabetes 2009; 10: 513-521.

27. Ersbøll M, Valeur N, Mogensen UM, et al . Global left ventricular longitudinal strain is closely associated with increased neurohormonal activation after acute myocardial infarction in patients with both reduced and preserved ejection fraction: A two-dimensional speckle tracking study. Eur J Heart Fail 2012; 14: 1121-1129. 
28. Hensel KO, Grimmer F, Jenke AC, Wirth S, Heusch A. The influence of real-time blood glucose levels on left ventricular myocardial strain and strain rate in pediatric patients with type 1 diabetes mellitus -a speckle tracking echocardiography study. BMC Cardiovasc Disord 2015; 15: 175.

29. van Heerebeek L, Hamdani N, Handoko ML, et al. Diastolic stiffness of the failing diabetic heart: Importance of fibrosis, advanced glycation end products, and myocyte resting tension. Circulation 2008; 117: 43-51.
30. van Heerebeek L, Somsen A, Paulus WJ. The failing diabetic heart: Focus on diastolic left ventricular dysfunction. Curr Diab Rep 2009; 9: 79-86.

31. Nakai H, Takeuchi M, Nishikage T, Lang RM, Otsuji Y. Subclinical left ventricular dysfunction in asymptomatic diabetic patients assessed by two-dimensional speckle tracking echocardiography: Correlation with diabetic duration. Eur J Echocardiogr 2009; 10: 926-932. 